\title{
Passive safety system for small unmanned aerial vehicles
}

\author{
Piotr Bartkowski ${ }^{1, *}$, Robert Zalewski ${ }^{1}$ \\ ${ }^{1}$ Institute of Machines Design Fundamentals, Warsaw University of Technology, Narbutta 84, 02-254 \\ Warsaw, Poland
}

\begin{abstract}
In this paper a new air-bag prototype suitable for protecting valuable objects mounted on the drone is presented. The paper provides a complimentary study involving both numerical simulations and experimental study. The experimental research results are presented for typical air-bag's textile material and were used as a base for the material model calibration process. This model was used for the numerical simulations of the proposed air-bag prototype, which were carried out in the LS-Dyna environment. Based on the outcome of the study, the proposed prototype seems to be a suitable device for preventing the unmanned vehicle equipment from unexpected accidents.
\end{abstract}

Keywords: Air-bag, drone, modelling, experiments, LS-Dyna, air-bag prototype

\section{Introduction}

Unmanned aerial vehicles (UAVs) have been dynamically developing during recent years, especially for commercial applications. UAVs are being used not only for the simplest tasks such as film recording or capturing pictures but also are used for more complex and responsible actions such as structure inspections or even to package delivering. Teleoperated multirotors are also used by the firefighters or police. In order to perform such operations, UAVs often require the installation of fragile, sensitive and very expensive additional equipment.

Statistics show that failures of the multirotors in the air and in consequences their crash with a ground are common. The collisions are very often caused by the components failure. Despite the fact that the design process and testing methodology have been strongly developed recently [1], it is technically impossible to eliminate all the accidents. According to [2] during the flight the most dangerous stage is landing. Although there are some landing systems available for manned airplanes, such as used at major airports Instrument Landing Systems (ILS), which significantly limit the number of crashes, they are not popular for unmanned aerial vehicles because of its complexity and weight. The landing processes of UAVs are therefore generally controlled manually or supported by more or less sophisticated portable external aiding systems [3].

\footnotetext{
* Corresponding author: bartkowski_piotr@onet.pl

Reviewers: Juraj Gerlici, Milan Nad'
} 
Another important issue is related to various mishaps occurring in the air, due to uncontrolled collisions with other flying or ground objects such as electric poles, transmission and telephone lines or even birds. Such situations generally lead to serious damages of UAVs and in consequences of the equipment attached to them. Despite the fact that various systems preventing air accidents are continuously improved and developed [4] there is still need to enhance their effectiveness.

An interesting solution reducing landing threats for UAVs is proposed in the paper [5], which presents a vision-based automatic landing system that uses a dome-shaped airbag. In the proposed solution the air-filled dome enables airplanes to land softly on the specially colored copula using advanced navigation sensors. Nevertheless, such a solution is insufficient, when the object was damaged in the air. Other interesting proposition for passive safety system was described in [6], which presents a parachute mounted on the UAV.

In this paper a new concept of passive safety system for unmanned aerial vehicles will be presented. The proposed solution consists of a special parachute and airbag attached to the UAV. The parachute protects the object in case of failure at high altitudes, whereas the airbag protects the device in case of damages just above the ground, where the parachute is ineffective. In this paper the authors focus on the design process of an air-bag. In subsequent sections the full concept of this innovatory system is presented in details.

\section{Material testing}

The concept of the discussed airbag was created with finite element method (FEM) using LS-DYNA environment. The most important stage in the airbag modeling process is calibrating a suitable material model, which must be backed up with experiments. The airbag is composed of a woven fabric which is rapidly inflated during a crash. For the analysis of the textile fabric it is necessary to perform a wide range of experiments. Beside the standard uniaxial tension test in various directions, it is necessary to conduct the shear test and additionally, what is highly recommended, biaxial tests. As far as the uniaxial tests is concerned, the experiment is relatively simple to carry out. Shear test and biaxial test are generally much more complex and generate more problems. Shearing tests are conducted in few different ways including bias extension test [7] or picture frame test [8,9]. Among popular biaxial test methodologies a hemisphere test is worth mentioning, biaxial test with cross-shape sample or inflated cylinder fabric test [10].

In this paper the results of simple uniaxial tests and bias extension tests of an airbag fabric PA 6.6 are presented. Samples used in uniaxial tests (Fig. 1) were stretched on MTS testing machine, however strains were measured by additional non-contact $3 \mathrm{D}$ deformation measuring system (ARAMIS). During bias extension tests the strains were measured based on the testing machine yaws displacement. Fig. 2 shows the typical results of the simple uniaxial tests. In Fig. 3 the results of bias extension tests are depicted. Additionally the photos recorded by the ARAMIS system including calculated strain maps are presented in Fig. 4. More detailed description of the fabric material laboratory tests can be found in the previous papers of the authors $[11,12]$.

The data acquired from the experiments were used in the next stage of modeling implemented to numerical model prepared in LS-Dyna software. This topic is described in details in the next part of this paper. 


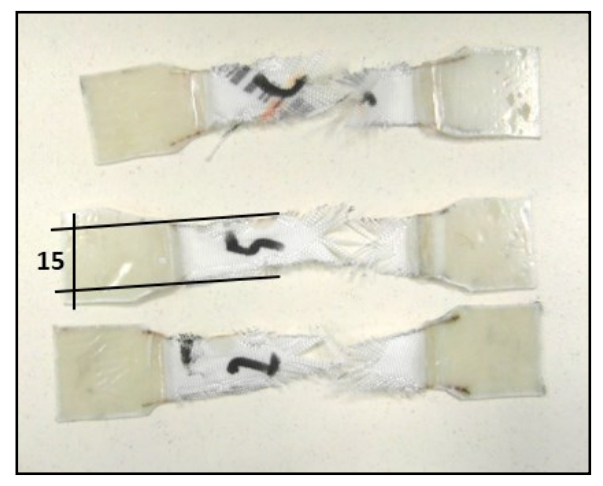

Fig. 1. Tested samples

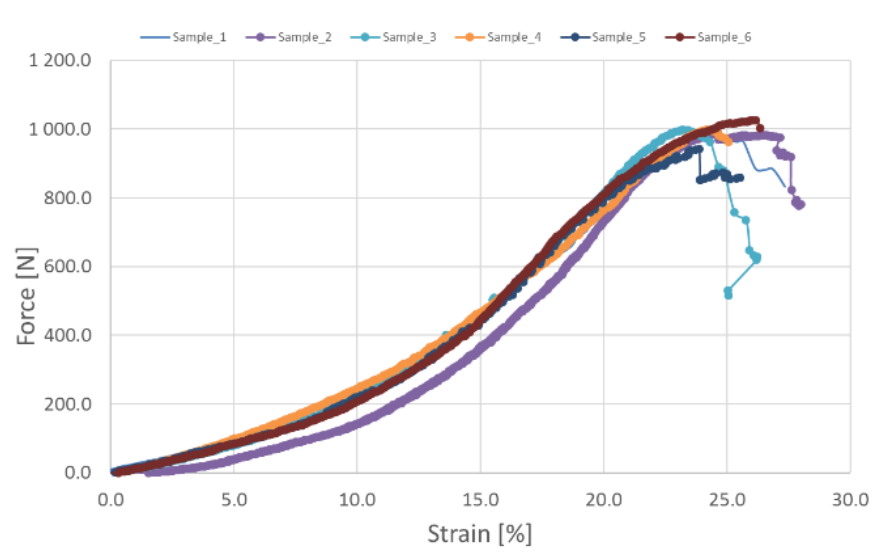

Fig. 2. Results of the uniaxial test

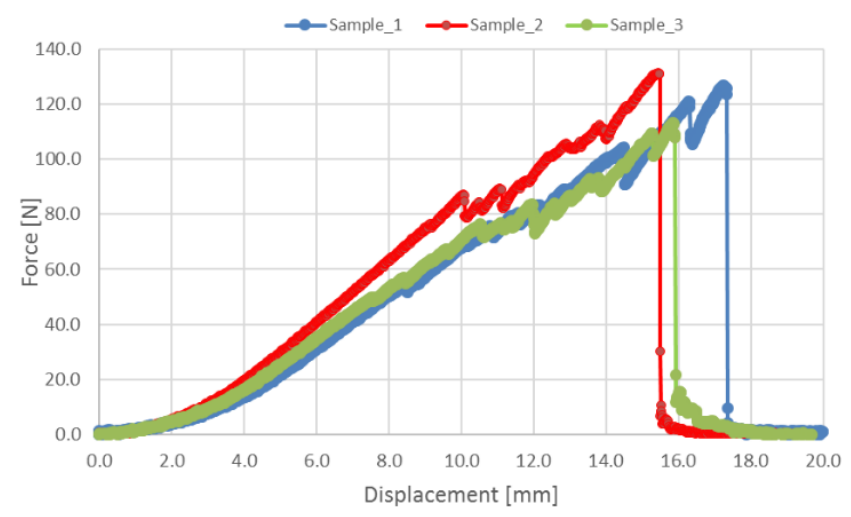

Fig. 3. Results of the bias extension test 


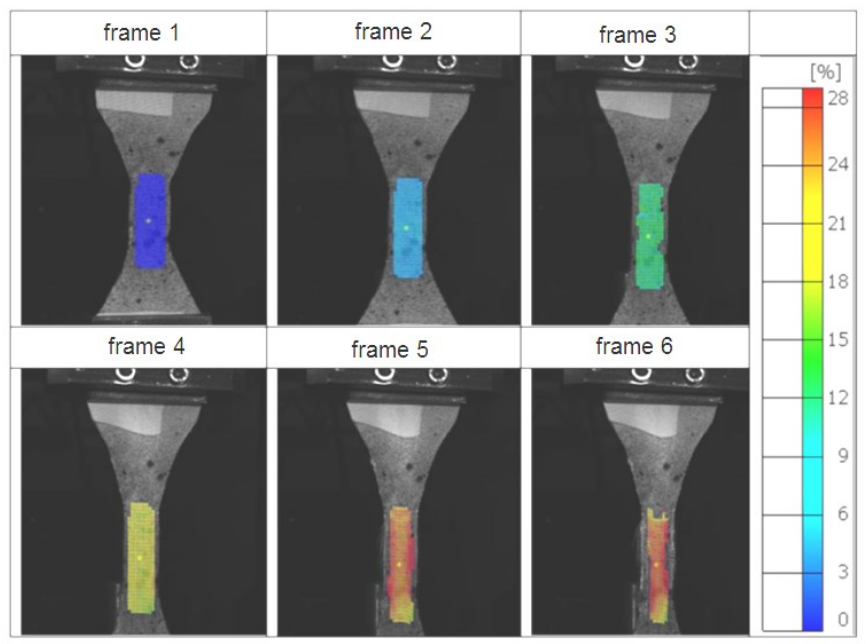

Fig. 4. Strain measured by ARAMIS system

\section{Experimental research of leakage behavior}

The crucial role in predicting the response of impacting object plays a gas leakage that is caused by fabric porosity or vents located on airbag surface (Fig. 5).

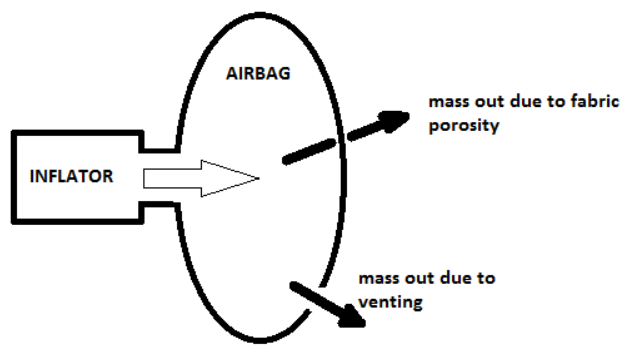

Fig. 5. Schematic illustration of the gas leakage

The modeling of leakage by vents is rather simple and will not be considered in this work. The more complicated task is modeling the leakage by material porosity. LS-DYNA provides various options for the airbag leakage modeling but the model must comply with the experiments. For this reason the airbag was tested on the drop test stand. During the experiments the internal pressure and strain of the airbag fabric were measured. The investigated airbag was filled up to predetermined pressure value and after that the intake of structure was closed. As a result the gas could only flow by fabric pores. The internal pressure was continuously measured during the outflow process. At the same time the average values of strains, calculated from the testing surfaces (Fig. 6) were recorded by the ARAMIS system. As the process of filling the air bag is rapid, the limitations of video measuring system (60 frames per second) did not allow to measure the strains during this stage. Therefore, the strains depicted in the Fig. 6 are equivalent to the maximum pressure reached in the air bag. 
Fig. 7 depicts the investigated airbag mounted on the test stand with strain maps calculated by the measuring system. In Fig. 8 variations of the internal pressure in time function are shown. Three different values of predefined maximal pressures were taken into consideration in laboratory tests. The obtained results were used in the model validation process to verify numerical results with experiments. Fig. 9 depicts the recorded experimentally characteristics of pressure in function of average strain determined from the area shown in Fig. 6. Fig. 10 confirms the reproducibility of the experimental results obtained from the experiments performed at a maximum pressure in the airbag.

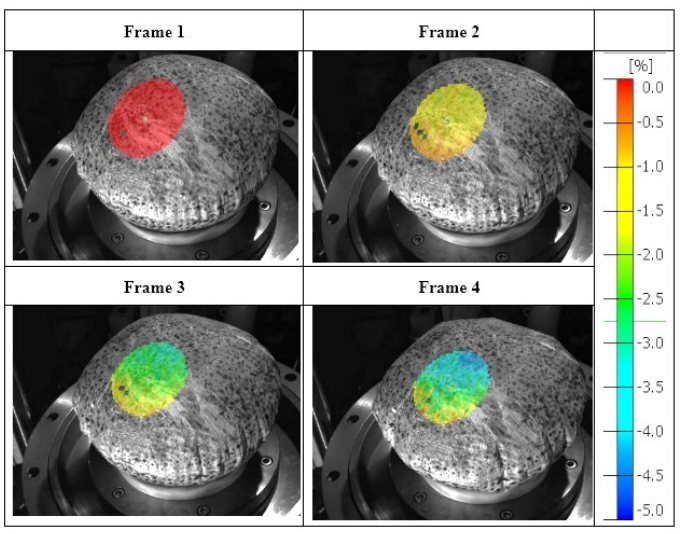

Fig. 6. Airbag strain maps

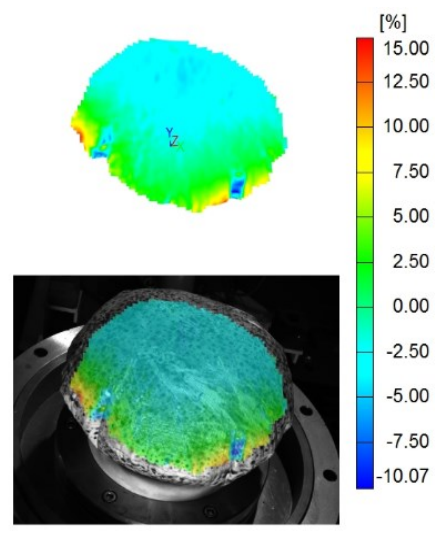

Fig. 7. Airbag with strain maps

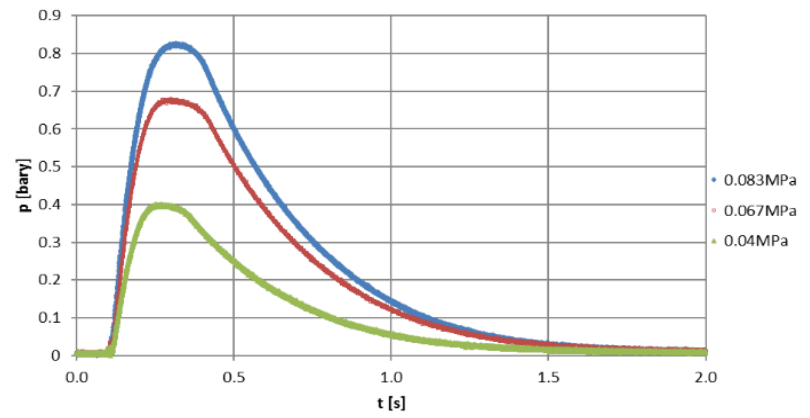

Fig. 8. Pressure in the airbag

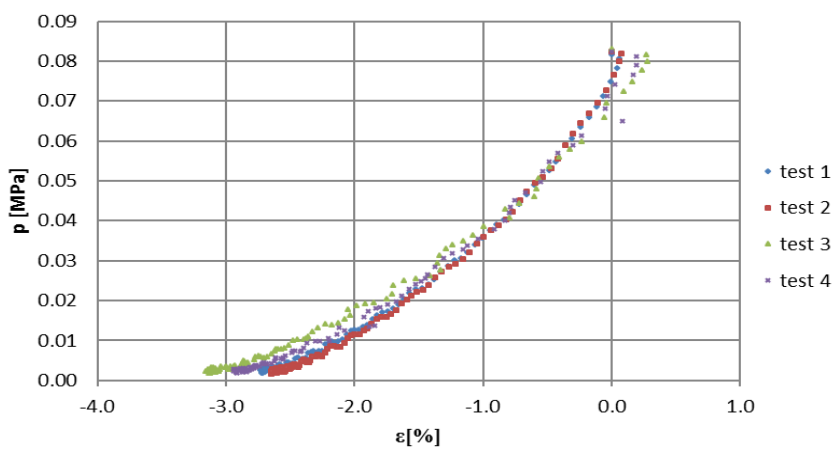

Fig. 9. Pressure in function of strain during unloading process 


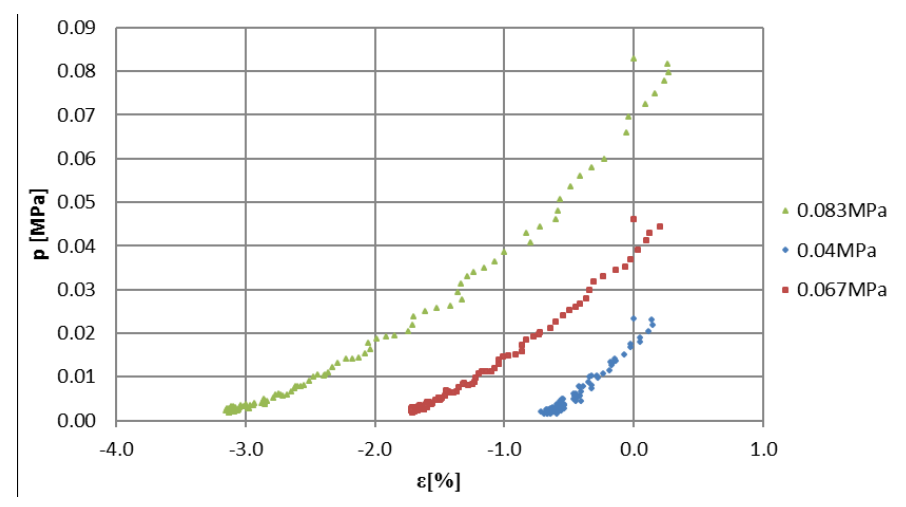

Fig. 10. Pressure in function of strain during unloading process

\section{Modeling of material mechanical behavior}

To model the real mechanical response of the considered fabric material in the LS-Dyna environment the material model MAT_FABRIC (\#34) was used [13]. This material model has been developed for many years beginning with the simple linear constitutive law and up to the latest implementation with a nonlinear biaxial loading and unloading curves. The newest model of the material allows to simulate the porosity of the structure where the gas flows out [14].

In order to ensure that the investigated airbag material model works correctly the comparison between simulation and experimental research was performed. Based on the geometrical dimensions of the tested samples FE models were prepared. Before the experimental data were implemented to FE model it was necessary to transform it into II Piola-Kirchhoff stress tensor and Green-Lagrange strain tensor. For the tension case it can be calculated from Eq. 1 and 2. Similarly, for shear tests it can be obtained from (3) and (4). The derivation of equations (1-4) is precisely described in paper $[11,12]$.

$$
\begin{gathered}
(\widetilde{\mathrm{T}})_{22}=\frac{\mathrm{f}}{\mathrm{A}_{0}} \cdot \frac{1}{1+\varepsilon_{\text {eng }}} \\
(\mathrm{E})_{22}=\frac{1}{2} \cdot\left(\mathbf{1}+\boldsymbol{\varepsilon}_{\text {eng }}\right)^{2}-\frac{1}{2} \\
(\widetilde{\mathrm{T}})_{21}=(\widetilde{\mathrm{T}})_{12}=\frac{\mathrm{f} \cdot\left(\mathrm{l}_{0}-\mathrm{W}\right)}{\mathrm{t} \cdot \mathrm{W}(\mathrm{lo}-\mathrm{W}+\mathrm{d})} \\
(\mathrm{E})_{12}+(\mathrm{E})_{21}=\left(1+\frac{\mathrm{d}}{\mathrm{l}_{\mathrm{o}}-\mathrm{W}}\right)^{2}-1
\end{gathered}
$$

Where $T_{22}, T_{21}, T_{12}$ - elements of stress tensor, $E_{22}, E_{21}, E_{12}$ - elements of strain tensor, $f$ - force measured by testing machine, $\varepsilon_{\text {eng }}$ - engineering strain, $A_{0}$ - sample cross section, $l_{o}$ initial length of the sample, $W$ - initial width of the sample, $t$ - thickness of the sample, $d$ yaws displacement.

When FE model was completed the analysis were performed using explicit LS-Dyna code. As a result it was possible to verify the experimental and numerical results. To ensure that the model correctly describes the stress and strain state in the material two independent 
tests were performed. The first one was related to the simple uniaxial tension test. In this probe the elongation of the sample corresponded to the main material direction. Forces in a strain function were determined numerically and in the final stage the results were compared with real experimental response of the material. As it is shown in Fig. 11 the model predicts precisely the behavior of the fabric.

In another experiment - bias extension test, the sample coordinate system was rotated by 45 degrees and the testing piece was again strained. In this case the observed differences between simulation and experimental results are more significant (Fig. 12). It is worth mentioning, that considering the real behavior of an airbag, the shear component in stress tensor does not play a significant role, consequently the authors assumed that model is reliable for further numerical analyses. Numerically calculated stress fields generated in loaded fabric samples in simple tensile test and bias extension test are depicted in Figs. 13 and 14 respectively.

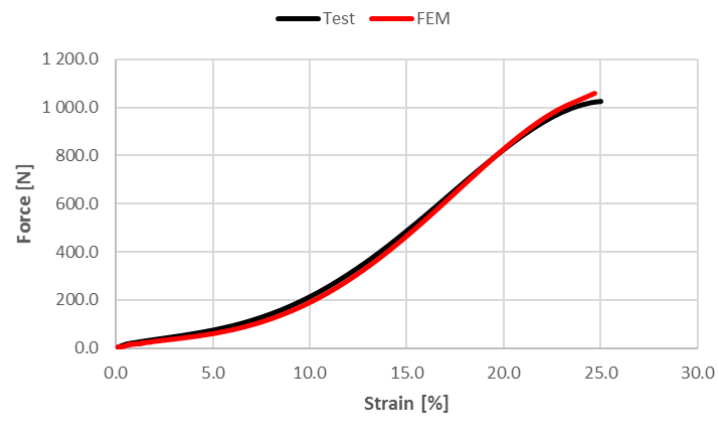

Fig. 11. Test vs experiments - simple uniaxial tension

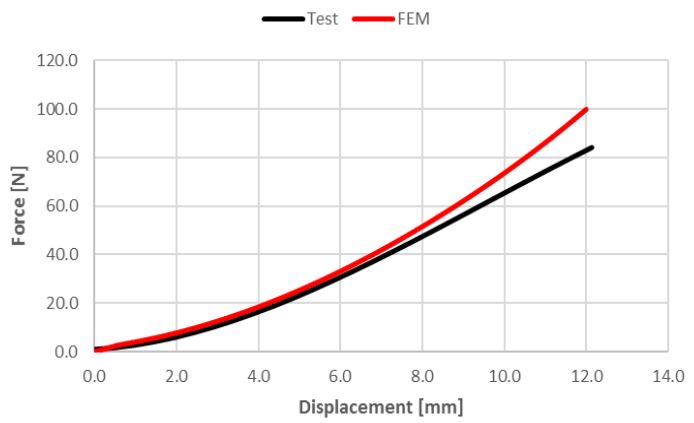

Fig. 12. Test vs experiments - bias extension test 


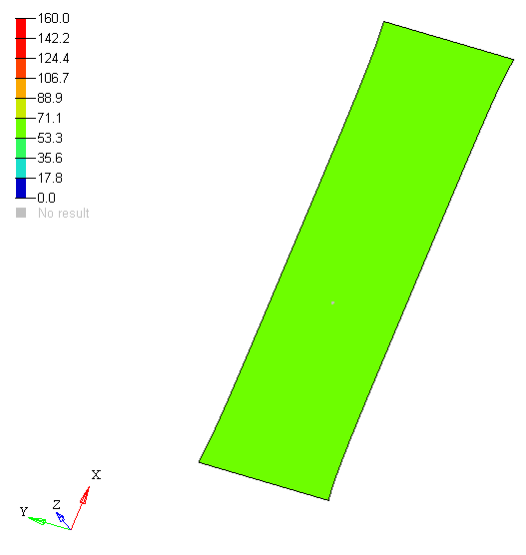

Fig. 13. Principal stress distribution in material sample - simple uniaxial tension

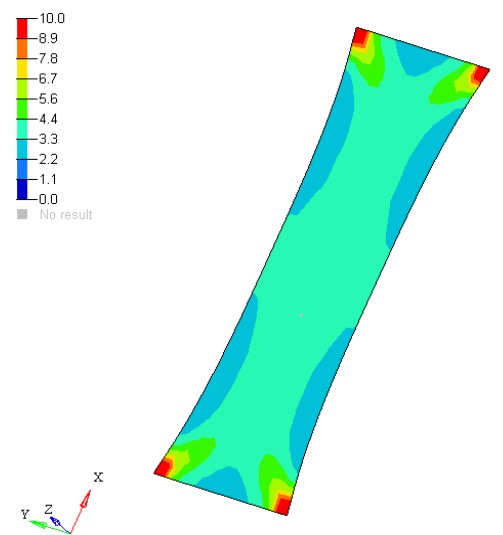

Fig. 14. Maximum shear stress distribution in material sample - bias extension test

\section{Leakage modeling}

Numerical evaluation of the leakage through the fabric involves computing the mass of the gas that needs to be removed from the airbag (Fig. 5). Primarly the leakage depends on three criteria: area of leakage, pressure and duration time. A more advanced model includes also blockage of the leakage area caused by the contact with an object. LS-DYNA offers few different formulations of the leakage caused by fabric porosity. In this paper WangNefske's formulas with blockage of the leakage area were used. Model is described by Eq. 5.

$$
\dot{\mathrm{m}}_{\text {out }}=\sqrt{\mathrm{n} \cdot \mathrm{g}_{\mathrm{c}}} \cdot\left[\sum_{1}^{\mathrm{n}}\left(\mathrm{FLC}(\mathrm{t})_{\mathrm{n}} \cdot \operatorname{FAC}(\mathrm{p})_{\mathrm{n}} \cdot \operatorname{Area}_{\mathrm{n}}\right)\right] \cdot \sqrt{2 \cdot \mathrm{p} \cdot \rho} \cdot \sqrt{\frac{\gamma \cdot\left(\mathrm{Q}^{\left.\frac{2}{\mathrm{k}}-\mathrm{Q}^{\frac{\gamma-1}{\gamma}}\right)}\right.}{\gamma-1}}
$$

Where: $g_{c}$ - gravitational conversion constant $\left[\frac{m}{s^{2}}\right], \mathrm{n}-1 \cdot \frac{s^{2}}{m}, \mathrm{FLC}(\mathrm{t})-$ material coefficient [-], FLC (p) - material coefficient [-], Area - fabric area $\left[\mathrm{m}^{2}\right], p$ - pressure [Pa], $\rho$ - density $\left[\mathrm{kg} / \mathrm{m}^{3}\right], \gamma-$ ratio of specific heats [-], $Q$ - pressure relation [-], $\dot{m}_{\text {out }}$ - mass ratio $[\mathrm{kg} / \mathrm{s}]$.

The model has two material coefficients $(\mathrm{FLC}(\mathrm{t}), \mathrm{FAC}(\mathrm{p}))$ that have to be identified experimentally. Those coefficients had a constant value equal 2160 and 0.0001 respectively. Measurement of the porosity coefficient can be a very difficult task, but several types of air permeability measuring instruments are available for general use in textile testing [9]. In general the idea involves controlled air flow by known fabric area at given pressure. In this paper a different approach was proposed. Experiments were performed on the real airbag. In the first step the investigated object was digitalized by reverse engineering method using Photomodeler. Afterward, the FE model was developed. The original concept of the gas leakage modeling process is presented in Fig. 15. 


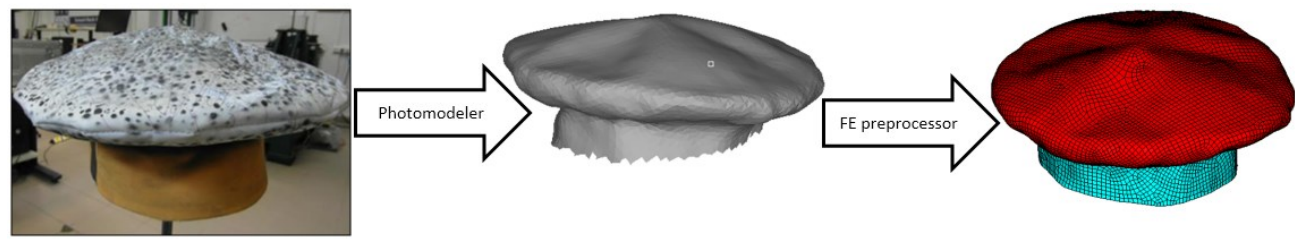

Fig. 15. Modeling approach

Fig. 16 presents FE airbag model on which the simulation process was based. The model calibration method consisted of changing iteratively the model coefficients to obtain the best compatibility between simulation and experiment. This procedure was performed for one selected initial condition (Fig. 17). When the model validation process was successfully completed, additional numerical experiments were carried out for other initial conditions that were also implemented in experimental procedure. Verification of the direct numerical data with laboratory tests results are shown in Figs. 18 and 19. As differences between numerical and experimental data are negligible, it can be assumed that the applied identification procedure is correct.

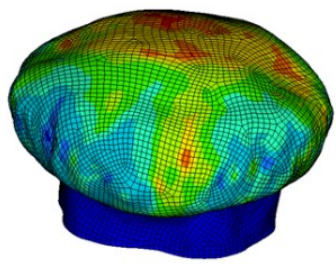

Fig. 16. FE model of airbag

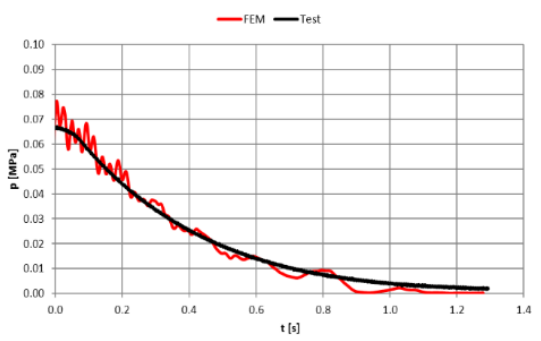

Fig. 18. Simulation vs test

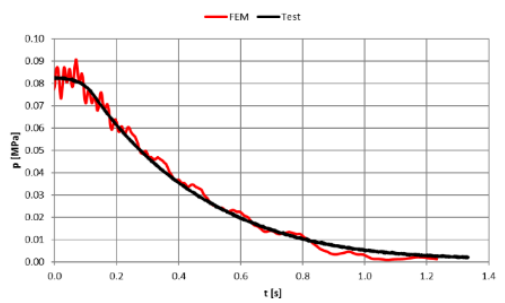

Fig. 17. Simulation vs test

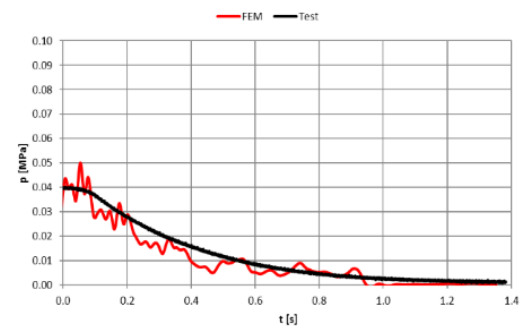

Fig. 19. Simulation vs test

\section{UAV airbag concept}

In the final stage of this paper the concept of UAV passive safety system will be described. The proposed system consists of two main parts, a parachute and an airbag. The purpose of applying the parachute is to protect UAVs in case of failure at high altitudes. The airbag is a suitable device when using parachute is impossible i.e. when the altitude of the object is lower than the threshold value. Fig. 20 shows the advantage of the proposed concept over the classical system including a simple parachute. Application of the additional airbag in the safety system for UAVs significantly reduces the hazardous altitudes range. 


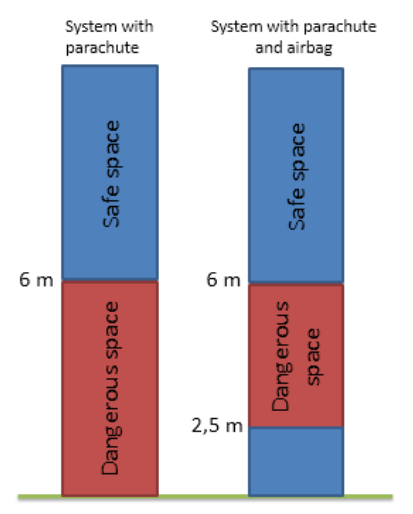

Fig. 20. Concept of airbag safety system

It is technically impossible to propose the system that protects multirotors independently on the accident location. It is assumed that the parachute is able to react when the mishap occurs on the highs over 6 meters. On the other hand it is technically impossible to consider the air-bag, which absorbs energy of the object falling from heights exceeding 6-meters because of the system mass.

Statistically most accidents occur close to the ground [2] so use of the airbag seems to be justified. The concept of the airbag was calculated with FEM applied in LS-DYNA software. It was assumed that the object crashes with the rigid obstacle at the 2.5 meters altitude, in consequences breaks down and begins to freely fall down. It was additionally assumed, that the drone is equipped with an additional device such as camera or other measuring system. The mass of the full system was estimated as $15 \mathrm{~kg}$. The main objective of the proposed passive safety system is to protect the valuable thermal imaging camera transported by the unmanned vehicle. Following pictures (Figs. 21-23) illustrate several subsequent stages of the numerical simulations. Fig. 24 depicts the acceleration in time variations of the object mounted on the investigated UAV. Based on the technical specifications provided by the camera producer the possible acceleration range is limited to $294,3 \frac{\mathrm{m}}{\mathrm{s}^{2}}$ only if it lasts not longer than $0.035 \mathrm{~s}$.

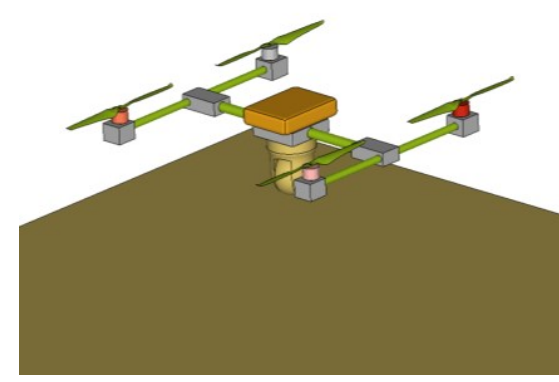

Fig. 21. Initial configuration of UAV and airbag - simulation time $=0$

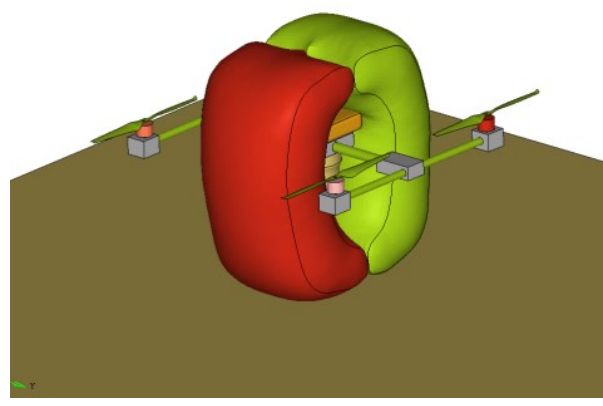

Fig. 22. Activation of the airbag - simulation time $=0.057 \mathrm{~s}$ 


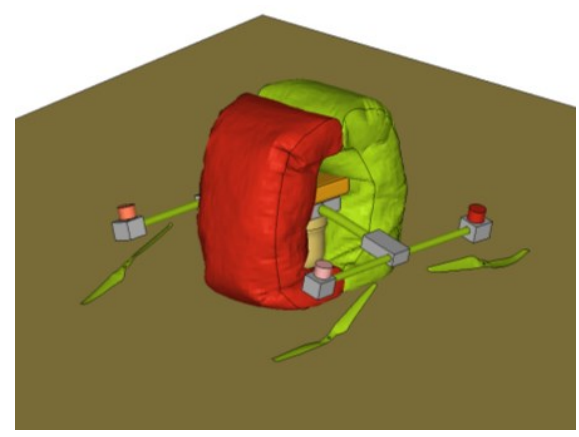

Fig. 23. Crash with the ground - simulation time $=0.12 \mathrm{~s}$

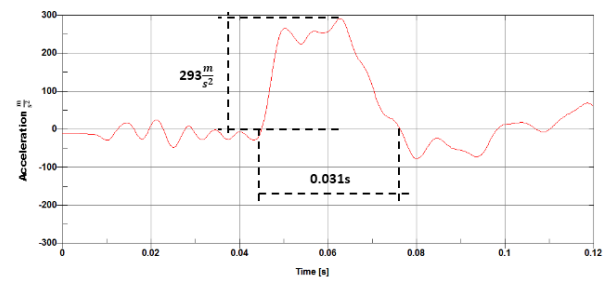

Fig. 24. Acceleration vs. time characteristics filter SAE 180

The unfolding process of the air bag was not considered in this paper. It was assumed that this process does not have a significant influence on the air bag behavior as well as for a whole system behavior. In order to improve the transparency of Fig. 21, the airbag was hidden.

\section{Conclusion}

In this paper the new concept of the safety system for UAVs was proposed, which combines the advantages of a parachute and the special airbag provided to protect an additional device transported by the flying object. The proposed concept seems to have a great potential for future engineering applications. In the paper not only the new concept of a passive safety system was presented but also the complex methodology that can be adopted for any design process for airbags was proposed. The conducted simulations revealed a technical capability of the proposed UAVs airbag project as all design assumptions were fulfilled. The project was developed based on the models verified with the experiments, what exactly fits in the contemporary way of design. It was shown how the mechanical properties of a textile material should be recorded and modeled. Furthermore, the original approach based on the reverse engineering method for the validation of the model leakage behavior was proposed.

The models of the drone and airbag proposed in this paper were to some extent simplified, as the real dynamics of such a complex rotating systems is very complicated. Consequently, the real behavior of the investigated system could be much more problematic. To fully confirm the correctness of the proposed approach to the discussed problem conducting more complex numerical simulations is required. In the final stage, the experimental research carried out on the real object is necessary to verify the proposed numerical approach.

\section{References}

1. C. Guowei, B. M. Chen, T. H. Lee, M. Dong, Design and implementation of a hardware-in-the-loop simulation system for small-scale UAV helicopters. Mechatronics 19, 1057-1066 (2009)

2. H. Sungsik, D. H. Shim, A vision-based landing system for small unmanned aerial vehicles using an airbag. Control Engineering Practice 18, 812-823 (2010) 
3. D. Fitzgerald, R. Walker, D. Campbell, A Vision Based Forces Landing Site Selection System for an Autonomous UAV. International Conference on Intelligent Sensors, Sensor Networks and Information Processing (2005)

4. M. Ptak, E. Rusinski, J. Karlinski, S. Dragan, Evaluation of kinematics of SUV to pedestrian impact-Lower leg impactor and dummy approach. Archives of Civil and Mechanical Engineering 12, 68-73 (2012)

5. H. Sungsik, D. H. Shim, A vision-based landing system for small unmanned aerial vehicles using an airbag. Control Engineering Practice 18, 812-823 (2010)

6. D.P. Hilliard, M. P. Hilliard, US 6338457 B1. US Patent (2002)

7. K. Potter, Bias extension measurements on cross-plied unidirectional prepreg. Compos. Part A. Appl. Sci. Manuf. 33, 63-73 (2002)

8. G.B. McGuinness, R.A. Canavan, T.A. Nestor, C.M. O’Bradaigh, A picture-frame intraply shearing test for sheet-forming of composite materials. Proceedings of ASME Materials Division 69, 1107-18 (1995)

9. G.B. McGuinness, C.M.O. Bradaigh, Development of rheological models for forming flows and picture-frame shear testing of fabric reinforced thermoplastic sheets. Journal of Non-Newtonian Fluid Mechanics 73, 1-28 (1997)

10. S. Farboodmanesh, J. Chen, Z. Tao, J. Mead, H. Zhang, Base fabrics and their interaction in coated fabrics. Smart Textile Coatings and Laminates, A volume in Woodhead Publishing Series in Textiles, 42-94 (2010)

11. P. Bartkowski, R. Zalewski, Experimental Research and Modeling of the Air-Bag Fabric Material. Machine Dynamics Research 39 (1), 143-152 (2015)

12. P. Bartkowski, R. Zalewski, Designing process of the drone's passive safety system. New Advances in Information Systems and Technologies, Á. Rocha et al. (eds.), Advances in Intelligent Systems and Computing 445, Springer International Publishing Switzerland, DOI 10.1007/978-3-319-31307-8_74, 729-737 (2016)

13. A. Hirth, A. Haufe, L. Olovsson, Airbag Simulation with LS-DYNA Past - PresentFuture. LS-DYNA Andwenderforum, Frankenthal (2007)

14. Schlenger, M., A New Model for Simulation of Fabric Leakage in LS-DYNA. LSDYNA Forum, Bamberg, D-III-7-22 (2010) 